\title{
Swing-Door Overlay Tympanoplasty: Surgical Technique and Outcomes
}

\author{
So Young Park · Hyuk Jae Lee $\cdot$ Myung Joo Shim $\cdot$ Dong Kee Kim · Byung Do Suh $\cdot$ Shi Nae Park \\ Department of Otorhinolaryngology-Head and Neck Surgery, College of Medicine, The Catholic University of Korea, Seoul, Korea
}

Objectives. The classical overlay tympanoplasty is technically difficult with some disadvantages and thus less popular. However, it is particularly useful for large, anterior perforations. In this study, we describe the technique of a modified overlay graft in the tympanoplasty coined as the swing-door overlay tympanoplasty and report its outcomes.

Methods. Retrospective review of patients undergoing the swing-door overlay tympanoplasty at a tertiary referral center between 2003 and 2016 was performed. Patient who had ossicular abnormality, previous tympanoplasty, and profound hearing loss were excluded. The surgical technique is described in detail. The outcomes were evaluated by the graft success rate, complication rate, and hearing results. The hearing level was determined by four pure-tone average at $0.5,1,2$, and $4 \mathrm{kHz}$. Air-bone gap closure was mainly assessed.

Results. A total of 306 patients (110 males and 196 females) were included. The mean age was $49.1 \pm 16.6$ years. Follow-up periods ranged from 6 to 108 months with an average of 18.4 months. The overall graft success rate reached $98.4 \%$. Five graft failures occurred with reperforation in three cases and lateralization in two cases. Postoperative complications occurred in 12 cases (3.9\%). Air-bone gap changes (closures) were $7.8 \pm 12.8,5.2 \pm 12.2,5.7 \pm 10.2$, and $6.0 \pm$ $12.8 \mathrm{~dB}$ at $0.5,1,2$, and $4 \mathrm{kHz}$, respectively (all $P<0.001$ ) with an average improvement of $6.2 \mathrm{~dB}$. Postoperative airbone gap was closed to $\leq 20 \mathrm{~dB}$ in $86.9 \%$.

Conclusion. The swing-door overlay tympanoplasty is a highly successful surgical technique suitable for all types of tympanic membrane perforations. This approach is technically easier than classical overlay tympanoplasty and affords an excellent graft success rate with satisfying hearing results.

Keywords. Tympanic Membrane Perforation; Temporal Fascia; Malleus; Onlay Tympanoplasty; Underlay Tympanoplasty

\section{INTRODUCTION}

Tympanoplasty, one of the most common ear surgeries, aims to eradicate the pathology and to restore the function of the middle ear in chronic otitis media. Intact tympanic membrane (TM) permits middle ear impedance matching and round window protection for effective sound transmission to the inner ear [1].

\footnotetext{
- Received December 14, 2017

Revised February 13, 2018

Accepted March 14, 2018

- Corresponding author: Shi Nae Park

Department of Otorhinolaryngology-Head and Neck Surgery, Seoul St.

Mary's Hospital, College of Medicine, The Catholic University of Korea,

222 Banpo-daero, Seocho-gu, Seoul 06591, Korea

Tel: +82-2-590-2215, Fax: +82-2-595-1354

E-mail: snparkmd@catholic.ac.kr
}

Upon these basic principles, the techniques have been developed through significant refinements over the decades since the term tympanoplasty was first introduced by Wullstein and Zollner in the early 1950s [1]. Practically, it is conservative to say that any of the otologic surgeon is ready to tailor the details in the tympanoplasty technique. Tympanoplasty is most commonly classified into two great divisions, overlay and underlay techniques, according to the placement of the graft relative to the fibrous annulus and denuded TM remnant. Comparatively easy underlay technique is popular, while more demanding overlay technique is reserved for total or anterior perforations, or failed surgery [2]. Each technique has its strengths and weaknesses. There could be risks of anterior blunting, lateralization, epithelial cyst, and delayed healing in the classical overlay technique. When an underlay graft is used, occasional fascia separation

Copyright @ 2018 by Korean Society of Otorhinolaryngology-Head and Neck Surgery.

This is an open-access article distributed under the terms of the Creative Commons Attribution Non-Commercial License (http://creativecommons.org/licenses/by-nc/4.0)

which permits unrestricted non-commercial use, distribution, and reproduction in any medium, provided the original work is properly cited. 
from the anterior annular shelf and resultant reperforation can make the underlay technique less suitable for large anterior perforations [2-4]. However, it should be noted that, regardless of the technique used, experienced surgeons achieve high success rates $[5,6]$. Another important consideration in the tympanoplasty is to choose the method to attach the graft to the malleus handle. The medial attachment, lateral attachment, and sandwich techniques have been documented [5]. Although the graft is commonly placed under the malleus to prevent lateralization, it can be placed lateral to the malleus, which acts as an additional point of support in the center of the graft $[2,7,8]$. Placing the fascia on the malleus is an advantage in cases with a retracted handle or denuded mucosa at the promontory [9]. The sandwich technique uses double fascia grafts, one medial and one lateral to the malleus to create an envelope around it, which can help secure its position $[5,10]$.

With the same questions as the majority of surgeons have about the necessity for removal of the canal skin in the classical overlay tympanoplasty, we have performed a modified overlay technique using Palva's swing-door flap introduced in the early 1960s [5,9]. This study was designed to describe the detailed technique of our swing-door overlay tympanoplasty and to report its surgical and functional outcomes.

\section{MATERIALS AND METHODS}

A retrospective review was conducted with the approval of the Institutional Review Board of Seoul St. Mary's Hospital, The Catholic University of Korea (IRB No. KC11RISE0853) on patients who underwent the swing-door overlay tympanoplasty by the author between 2003 and 2016 at our institution, a tertiary referral center, and were followed up for at least 6 months postoperatively. Patient who underwent ossiculoplasty, mastoidectomy, or revision tympanoplasty, and those with profound hearing loss were excluded from the study. Surgical outcomes were evaluated by the graft take and complication rates. The graft take meant complete perforation closure without TM lateralization or anterior angle blunting. Functional outcomes were assessed using pre- and postoperative (the last) audiograms. Air conduc-

\section{H I G H L I G G H T S}

- Our modified overlay technique uses swing-door flap without removal of the canal skin.

- Swing-door overlay tympanoplasty provided a graft success rate of $98.4 \%$.

- The overall air-bone gap closure was $6.2 \mathrm{~dB}$ after the swingdoor overlay tympanoplasty.

- Postoperative air-bone gap was closed to $\leq 20 \mathrm{~dB}$ in $86.9 \%$ of patients. tion (AC) and bone conduction (BC) pure-tone averages (PTAs) were calculated using 0.5-, 1-, 2-, and 4-kHz thresholds, and rounded to the nearest whole number. Air-bone gap (ABG) was defined as the difference between the $\mathrm{AC}$ and $\mathrm{BC}$ at each frequency. The outcome measures included (1) the graft success rate, (2) ABG change, i.e., the number of decibels of ABG closure (preoperative ABG minus postoperative ABG), (3) the proportion of patients in the ABG bins (0-10 dB, 11-20 dB, 21-30 $\mathrm{dB}$, and $>30 \mathrm{~dB}$ ), and (4) the complication rate. To compare the pre- and postoperative audiometric data, statistical analysis was performed using SPSS ver. 18.0 (SPSS Inc., Chicago, IL, USA) at a two-tailed significance level of 0.05 .

\section{Surgical technique}

The operations were performed under general endotracheal anesthesia. The postauricular approach was used and the temporalis fascia was harvested for grafting in all patients. A curvilinear incision was made along the posterior canal skin approximately $7 \mathrm{~mm}$ lateral to the annulus. A tympanomeatal flap was elevated from the posterior canal wall down to the annulus, preserving the fibrous annulus at its bony sulcus (Fig. 1A). The flap was then cut in a vertical direction at the 9-o'clock position, and divided into superior and inferior flaps. The TM remnant was dissected off the malleus handle and removed almost entirely to provide a fresh rim for the graft (Fig. 1B). Superiorly and inferiorly based swing-door flaps were rotated anteriorly, which permits good view of the entire tympanum and thus facilitates the removal of the pathologic tissues in the middle ear as well as fascia grafting. A laterally based anterior meatal flap was elevated, leaving the anterior annulus intact (Fig. 1C). After the dried fascia was trimmed to the suited size, an S-shaped slit was made at the anterosuperior edge (Fig. 1D). The fascia graft was placed over the anterior fibrous annulus and medial to the handle of malleus, and draped up the posterior canal wall. More concretely, the inferior part of the slit fascia was tucked underneath the malleus, and the convex anterior and concave posterior limbs in the superior part were pulled up over the malleus in a sandwichlike fashion of fascia-handle-fascia. All canal flaps were replaced over the fascia graft (Fig. 1E). The anterior tympanomeatal angle was firstly packed with several small pieces of antibiotic-impregnated gauze strips to secure the fascia-flap combination to the annulus and canal wall, and also to prevent blunting. The fascia and flap were fixed in the canal with bigger pledgets (Fig. 1F).

\section{RESULTS}

A total of 306 patients undergoing the swing-door overlay tympanoplasty were eligible for the study (110 males and $196 \mathrm{fe}$ males). The mean age was 49 years (range, 5 to 80 years). Follow-up periods ranged from 6 months to 9 years with a mean of 18.4 months. The perforations were variable in site and size. The 


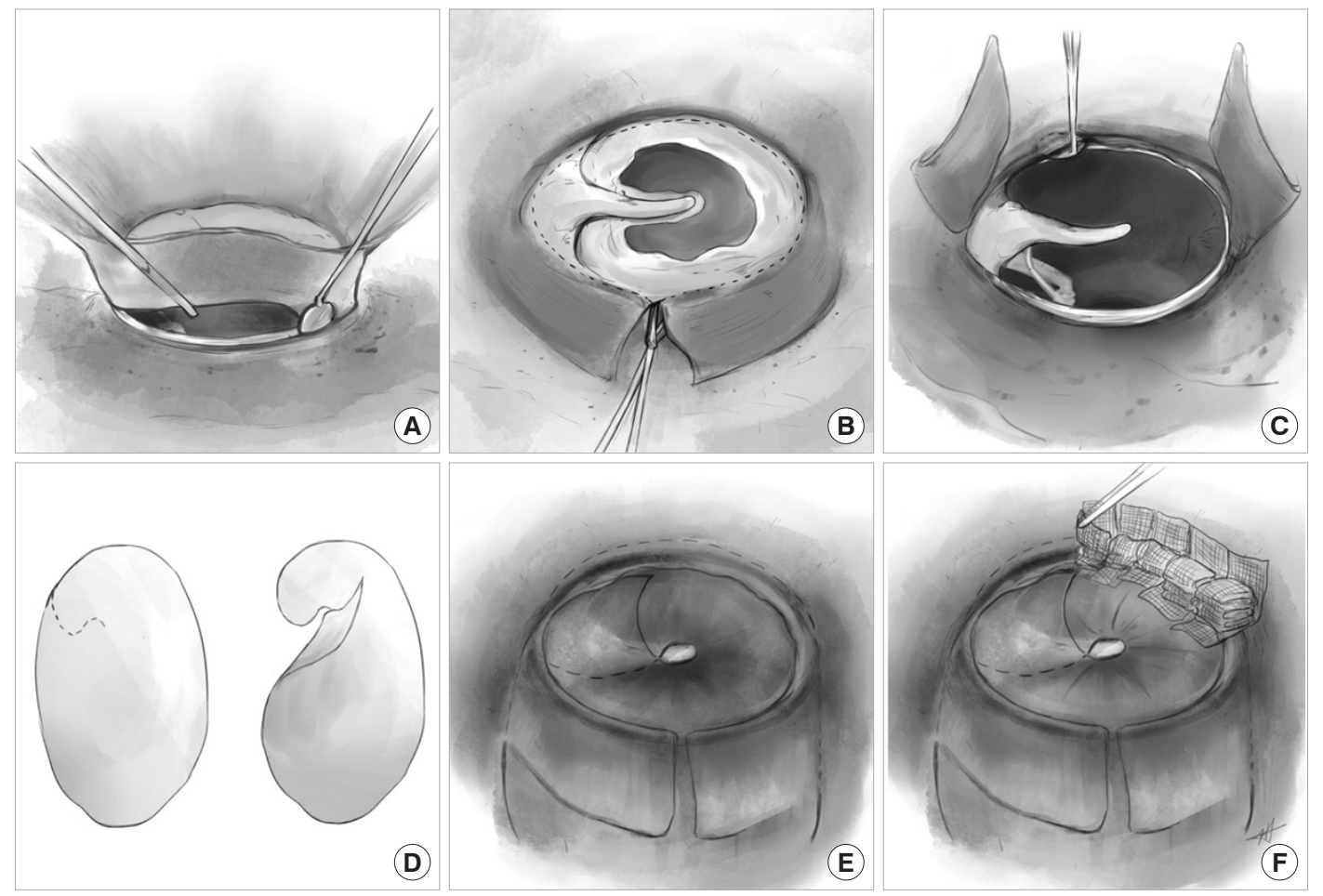

Fig. 1. Surgical technique of the swing-door overlay tympanoplasty. (A) A posterior tympanomeatal flap is elevated down to the annulus with the fibrous annulus preserved at its bony sulcus. (B) The flap is cut in a vertical direction at the 9-o'clock position, and divided into superior and inferior flaps. The drum remnant is dissected off the malleus handle and removed almost entirely. (C) Superiorly and inferiorly based swing-door flaps are rotated anteriorly. Laterally based anterior meatal flap is elevated to expose the anterior annulus. (D) The dried temporalis fascia is trimmed to the right size. An S-shaped slit is made at the anterosuperior edge. (E) The fascia is placed over the fibrous annulus and medial to the malleus handle in a sandwich-like fashion. All canal flaps are replaced over the fascia graft. (F) The anterior tympanomeatal angle is packed with several pieces of antibiotic-impregnated gauze strips, securing the fascia and flap to the annulus and canal wall.

Table 1. Clinical data of the study subjects

\begin{tabular}{lc}
\hline Variable & Value $(\mathrm{n}=306)$ \\
\hline Age $(\mathrm{yr})$ & $49.1 \pm 16.6(5-80)$ \\
Male & $46.6 \pm 16.9$ \\
Female & $50.5 \pm 16.3$ \\
Sex (male:female) & $110: 196$ \\
Involved ear (right:left) & $152: 154$ \\
Site of perforation & \\
Central & $164(53.6)$ \\
Anterior & $87(28.4)$ \\
Posterior & $29(9.5)$ \\
Marginal & $26(8.5)$ \\
AC PTA (dB) & $35.1 \pm 17.0$ \\
BC PTA (dB) & $15.3 \pm 14.7$ \\
ABG (dB) & \\
$0.5 \mathrm{kHz}$ & $19.7 \pm 14.1$ \\
$1 \mathrm{kHz}$ & $20.3 \pm 11.3$ \\
$2 \mathrm{kHz}$ & $14.8 \pm 9.6$ \\
$4 \mathrm{kHz}$ & $24.5 \pm 12.6$ \\
Average & $19.8 \pm 9.4$ \\
\hline
\end{tabular}

Values are presented as mean \pm standard deviation (range), mean \pm standard deviation, or number (\%).

AC, air conduction; PTA, pure-tone average; $B C$, bone conduction; $\mathrm{ABG}$ air-bone gap.
Table 2. Postoperative complications

\begin{tabular}{lc}
\hline Variable & Patient $(n=306)$ \\
\hline Reperforation & $3(0.98)$ \\
Lateralization & $2(0.65)$ \\
Anterior angle blunting & 0 \\
Keratin pearl or cyst & $7(2.29)$ \\
Infection or myringitis & 0 \\
\hline
\end{tabular}

Values are presented as number (\%).

detailed clinical features and preoperative audiometric data are presented in Table 1. Complete perforation closure without lateralization or anterior angle blunting was achieved in 301 among 306 patients. The graft success rate was as high as $98.4 \%$. Postoperative complications occurred in 12 cases (3.9\%): reperforation (three cases), lateralization (two cases), and epithelial cyst (seven cases). There was no anterior blunting or myringitis (Table 2). Representative pre- and postoperative TM findings of a few cases are demonstrated in Fig. 2. Pre- and postoperative AC PTAs were $35.1 \pm 17.0$ and $30.4 \pm 17.5 \mathrm{~dB}$ with significant improvement after the surgery $(P<0.001)$ (Fig. 3$)$. The mean ABGs were reduced by 7.8, 5.2, 5.7, and $6.0 \mathrm{~dB}$ at $0.5,1,2$, and $4 \mathrm{kHz}$, respectively (all $P<0.001$ ) (Table 3). Before the surgery, 

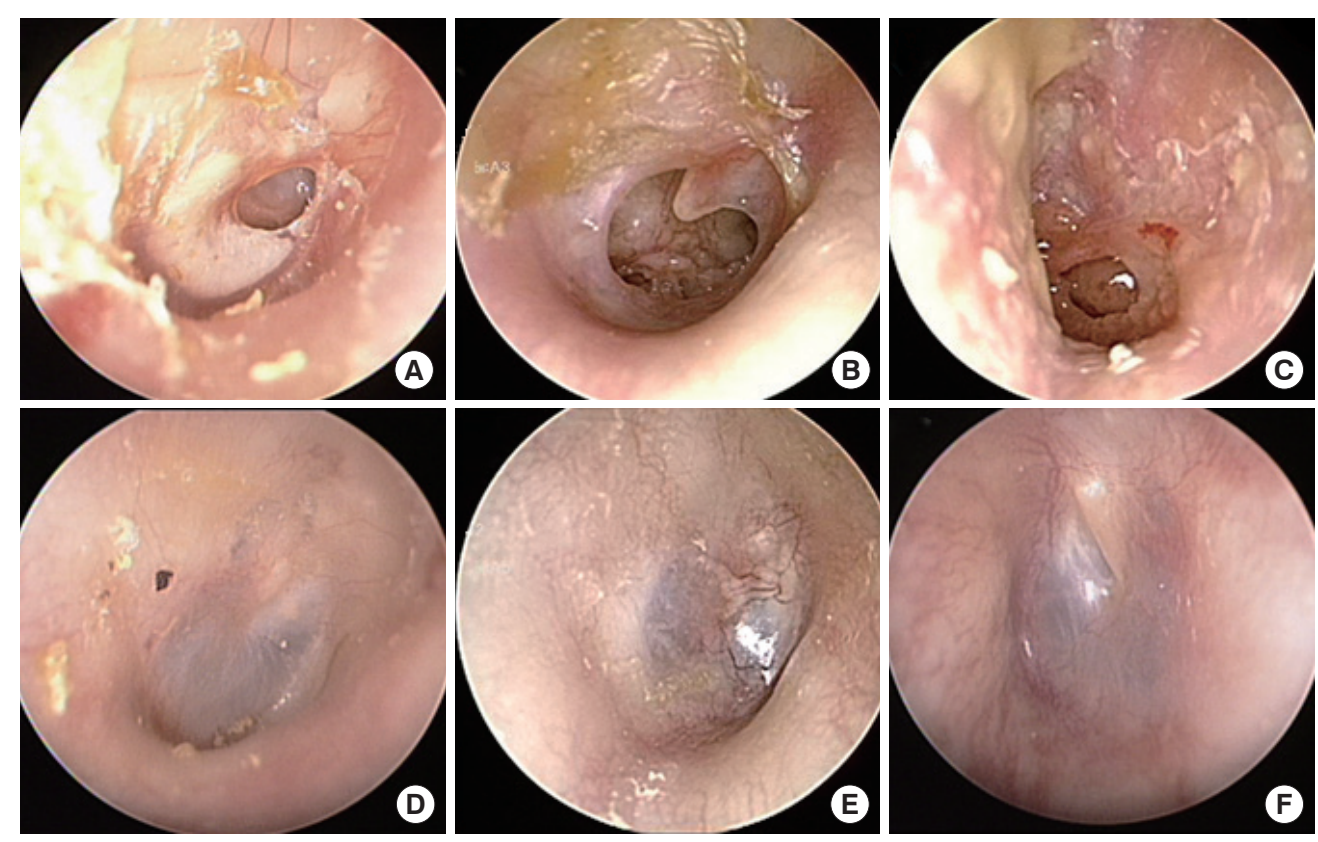

Fig. 2. Representative endoscopic findings of three tympanic membranes before (A-C) and after (D-F) the swing-door overlay tympanoplasty.
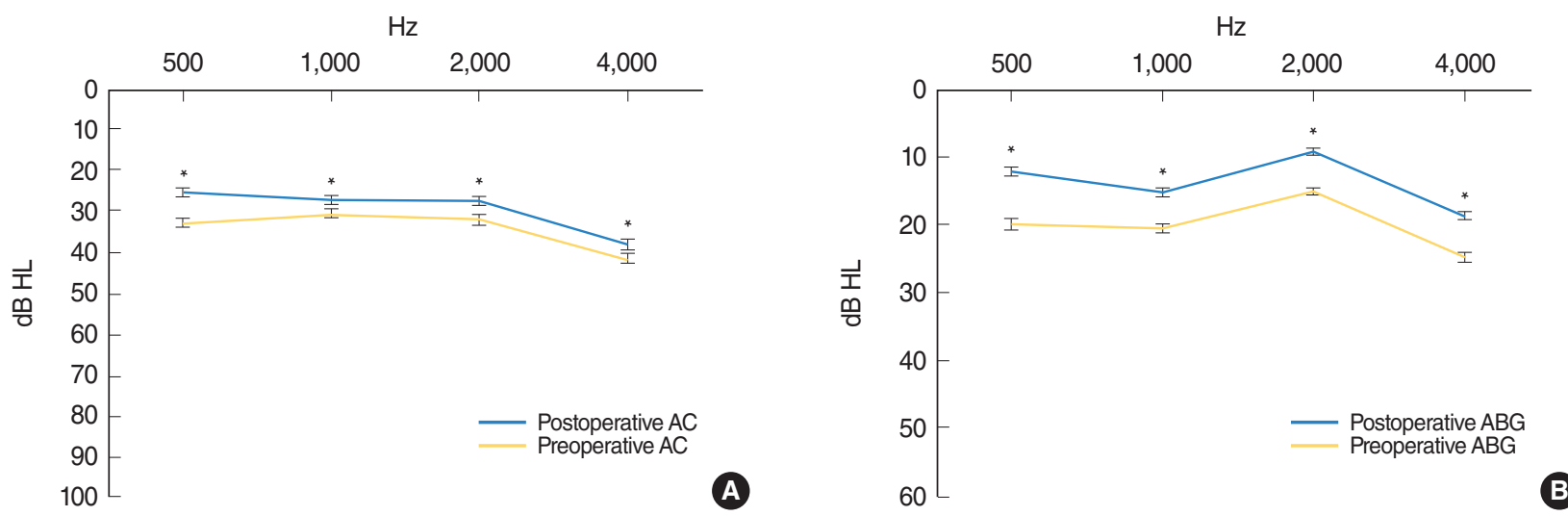

Fig. 3. (A) Pre- and postoperative mean air conduction (AC) thresholds at four frequencies in patients undergoing the swing-door overlay tympanoplasty. (B) Pre- and postoperative mean air-bone gaps (ABGs). HL, hearing level. ${ }^{*} P<0.001$, vs. preoperative value, paired $t$-test $(n=306)$. Error bars indicate standard error of mean.

Table 3. Hearing results of the swing-door overlay tympanoplasty

\begin{tabular}{ll}
\hline Variable & \multicolumn{1}{c}{ Value } \\
\hline Postoperative AC PTA $(\mathrm{dB})$ & $30.4 \pm 17.5$ \\
Postoperative BC PTA $(\mathrm{dB})$ & $16.8 \pm 15.8$ \\
Postoperative ABG $(\mathrm{dB})$ & $13.6 \pm 7.5$ \\
Change in ABG $(\mathrm{dB})^{\mathrm{a})}$ & \\
$0.5 \mathrm{kHz}$ & $7.8 \pm 12.8$ \\
$1 \mathrm{kHz}$ & $5.2 \pm 12.2$ \\
$2 \mathrm{kHz}$ & $5.7 \pm 10.2$ \\
$4 \mathrm{kHz}$ & $6.0 \pm 12.8$ \\
Average & $6.2 \pm 9.2$ \\
\hline
\end{tabular}

Values are presented as mean \pm standard deviation.

$A C$, air conduction; PTA, pure-tone average; $B C$, bone conduction; $A B G$ air-bone gap; Change in $A B G$, preoperative $A B G$-postoperative $A B G$. a) All $P<0.001$, paired t-test.
131 patients (42.8\%) had an ABG of $>20 \mathrm{~dB}$. After the surgery, 117 patients $(38.2 \%)$ showed an $\mathrm{ABG}$ of $\leq 10 \mathrm{~dB}$ and 149 patients (48.7\%), an ABG of 11-20 dB. Of the 306 patients, 266 $(86.9 \%)$ acquired an $\mathrm{ABG}$ of $\leq 20 \mathrm{~dB}$ postoperatively. The change in $\mathrm{ABG}$ distribution after the surgery was statistically significant $(P<0.001)$ (Fig. 4).

\section{DISCUSSION}

In this study, we described the surgical technique of the swingdoor overlay tympanoplasty and reported its outcomes in a large group of patients. To obtain a thin, conically shaped, vibrating membrane, the choice of an optimal technique of tympano- 


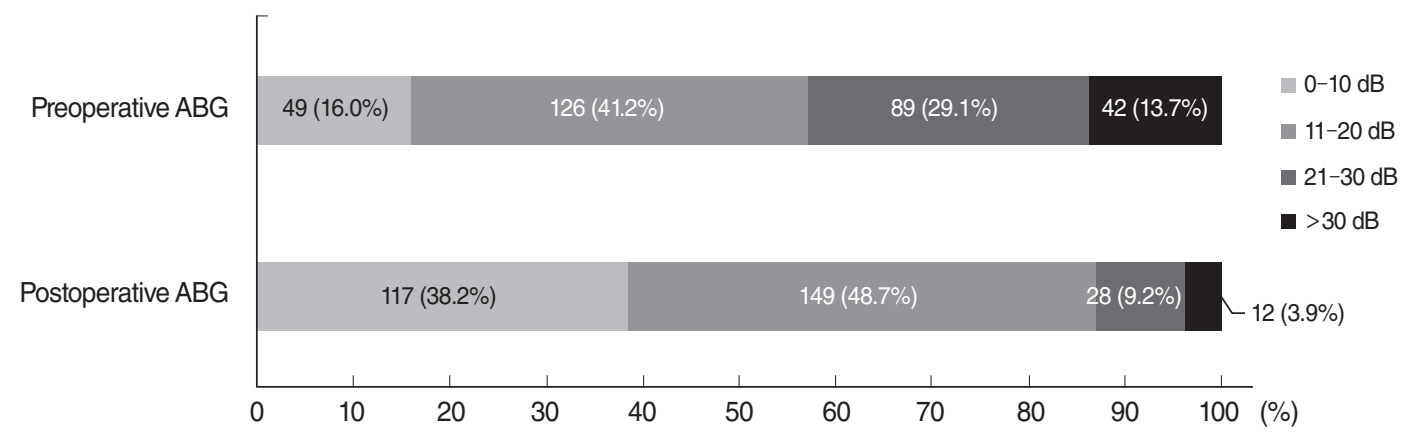

Fig. 4. Pre- and postoperative distribution of patients in the air-bone gap (ABG) bins with significant difference $(n=306 ; P<0.001$, chi-square test).

plasty is important. It usually depends on the surgeon's preference and skills, and not on the type of the TM perforation [11]. Nardone et al. [12] documented that the overlay technique offered a higher success rate than the underlay technique. It was explained by better exposure of the anterior sulcus and the preservation of the fibrous annulus with a two-fold vascularization in the overlay technique. The anteriorTM has less vascularity than the posterior TM. Applebaum and Deutsch [13] demonstrated that the posterior half of the TM is consistently better perfused through the mallear artery than the anterior half supplied by branches from the annular ring of blood vessels. On the other hand, Sergi et al. [11] reported that the underlay technique provided slightly higher graft healing rate and a significantly better hearing gain than the overlay technique. Nonetheless, they recommended the overlay technique in cases of larger and anterior perforations. Residual perforations always occurred in the anterior TM in the underlay technique, and a longer healing time was observed in the overlay technique [11]. Our modified overlay tympanoplasty keeps the advantage and supplements the weakness of the overlay tympanoplasty. The overlay technique ensures more secure placement of the graft on the annular shelf. Our key modifications to the classical overlay tympanoplasty include (1) the lift and replacement of the tympanomeatal flap without removal of the canal skin, (2) the swing-door flap adopted from Palva's swing-door underlay tympanoplasty $[5,9]$, which provides a good view of the entire middle ear, and (3) the S-shaped slit on the fascia, which facilitates sandwich-like attachment of the graft to the malleus to prevent lateralization. In addition, we suggest that (1) complete removal of the diseased TM remnant helps to attain the goal of a dried neodrum and (2) firm packing of the anterior annulus contributes to maintaining the sharp angle of the anterior wall.

An excellent graft success rate of $98.4 \%$ was achieved by the swing-door overlay tympanoplasty for all types of TM perforations. Our result is consistent with those of various tympanoplasties. Jung and Park [4] reported a 97\% success rate using mediolateral graft tympanoplasty; Schwaber [5], 95\% with a modified swinging door underlay technique; Farrior [10], 98\% using sandwich graft tympanoplasty; Schraff et al. [14], 94.5\% using window shade tympanoplasty; Ryan and Briggs [15], 98.7\% with the classical overlay graft technique; Peng and Lalwani [16], 96\% using hammock tympanoplasty; Shim et al. [17], $93.2 \%$ using three-point fix tympanoplasty. When the surgery is performed by a skilled and experienced otologic surgeon, the graft success rate is invariably high $[10,15]$. Postoperative complication rate was $3.9 \%$. There were no cases of anterior sulcus blunting in spite of the fact that the graft was placed over the anterior annulus. Epithelial cyst occurred most commonly, which was removed easily using a pick on an outpatient basis during the follow-up period. In contrast to the surgical outcomes, functional outcomes in the previous literature have been relatively variable according to the different measures and cohorts. In this study, a significant improvement was observed in the AC PTA postoperatively. The overall ABG closure was 6.2 $\mathrm{dB}$ at an average follow-up of 18.4 months, which is very similar with the results reported by other investigators. Kartush et al. [2] reported a $5.3 \mathrm{~dB}$ average improvement in ABG using over-under tympanoplasty; Peng and Lalwani [16], $7.8 \mathrm{~dB}$ using hammock tympanoplasty. To explain the relatively small ABG changes, preoperative hearing is worth noting. Subjects in this study had mild conductive loss ( $35 \mathrm{~dB}$ AC PTA with $20 \mathrm{~dB}$ ABG) because patients with ossicular abnormalities were excluded. Postoperative ABG was closed to $\leq 20 \mathrm{~dB}$ in $86.9 \%$ of cases, which is comparable to other studies. Ryan and Briggs [15] reported a total of $82.5 \%$ of cases with a postoperative ABG of less than $20 \mathrm{~dB}$ with the classical overlay graft technique; Shim et al. [17], 76.4\% using three-point fix tympanoplasty. Schraff et al. [14] reported a higher rate of $98 \%$ closure of the ABG within $20 \mathrm{~dB}$ using window shade tympanoplasty. ABG of $>20 \mathrm{~dB}$ in 40 patients (13.1\%) may be explained by (1) a delayed recovery of the Eustachian tube function due to relatively short follow-up periods in this study or (2) pre-existing Eustachian tube dysfunction in those patients. Our results suggest that this modified overlay tympanoplasty using the swingdoor flap is an effective procedure which provides a very high graft success rate and good hearing outcomes.

In conclusion, the swing-door overlay tympanoplasty is a highly successful technique suitable for all types of TM perfora- 
tions. This approach is easy to perform and affords an excellent graft success rate with satisfying hearing results. Few complications are additional strengths.

\section{CONFLICT OF INTEREST}

No potential conflict of interest relevant to this article was reported.

\section{ACKNOWLEDGMENTS}

We would like to thank Ms. Hyo Jeong Yang, for the illustrations of the surgical technique used in this article.

\section{REFERENCES}

1. Briggs RJ, LuxfordWM. Chronic ear surgery: a historical review. Am J Otol. 1994 Jul;15(4):558-67.

2. Kartush JM, Michaelides EM, Becvarovski Z, LaRouere MJ. Overunder tympanoplasty. Laryngoscope. 2002 May;112(5):802-7.

3. Farrior JB. The anterior tympanomeatal angle in tympanoplasty: surgical techniques for the prevention of blunting. Laryngoscope. 1983 Aug;93(8):992-7.

4. Jung TT, Park SK. Mediolateral graft tympanoplasty for anterior or subtotal tympanic membrane perforation. Otolaryngol Head Neck Surg. 2005 Apr;132(4):532-6.

5. Schwaber MK. Postauricular undersurface tympanic membrane grafting: some modifications of the "swinging door" technique. Otolaryngol Head Neck Surg. 1986 Sep;95(2):182-7.

6. Rizer FM. Overlay versus underlay tympanoplasty. Part I: historical review of the literature. Laryngoscope. 1997 Dec;107(12 Pt 2):1-25.

7. Stage J, Bak-Pedersen K. Underlay tympanoplasty with the graft lateral to the malleus handle. Clin Otolaryngol Allied Sci. 1992 Feb;17(1):6-9.

8. Yawn RJ, Carlson ML, Haynes DS, Rivas A. Lateral-to-malleus underlay tympanoplasty: surgical technique and outcomes. Otol Neurotol. 2014 Dec;35(10):1809-12.

9. Tos M. Underlay techniques. In:Tos M, editor. Manual of middle ear surgery. Vol 1. New York (NY): Thieme Medical Publishers; 1993. p. 188-94.

10. Farrior JB. Sandwich graft tympanoplasty: experience, results, and complications. Laryngoscope. 1989 Feb;99(2):213-7.

11. Sergi B, Galli J, De Corso E, Parrilla C, Paludetti G. Overlay versus underlay myringoplasty: report of outcomes considering closure of perforation and hearing function. Acta Otorhinolaryngol Ital. 2011 Dec;31(6):366-71.

12. Nardone M, Sommerville R, Bowman J, Danesi G. Myringoplasty in simple chronic otitis media: critical analysis of long-term results in a 1,000-adult patient series. Otol Neurotol. 2012 Jan;33(1):48-53.

13. Applebaum EL, Deutsch EC. An endoscopic method of tympanic membrane fluorescein angiography. Ann Otol Rhinol Laryngol. 1986 Sep-Oct;95(5 Pt 1):439-43.

14. Schraff S, Dash N, Strasnick B. "Window shade" tympanoplasty for anterior marginal perforations. Laryngoscope. 2005 Sep;115(9):16559.

15. Ryan JE, Briggs RJ. Outcomes of the overlay graft technique in tympanoplasty. ANZ J Surg. 2010 Sep;80(9):624-9.

16. Peng R, Lalwani AK. Efficacy of "hammock" tympanoplasty in the treatment of anterior perforations. Laryngoscope. 2013 May;123(5): 1236-40.

17. Shim DB, Kim HJ, Kim MJ, Moon IS. Three-point fix tympanoplasty. Acta Otolaryngol. 2015 May;135(5):429-34. 